


\title{
Cicloturismo en Costa Rica: una mirada a los tour operadores
}

\author{
Cycle tourism in Costa Rica: a perspective from the current tour operators \\ Carlos Eduardo Ledezma Benavides ${ }^{1}$ \\ Recibido: 25/8/2016 / Aprobado:1/11/2016
}

\begin{abstract}
Resumen
La presente investigación, tiene como objetivo describir la situación actual del cicloturismo en Costa Rica, desde la perspectiva de los tour operadores que ofertan la actividad en el país, al valorar las características propias del tour: fortalezas, debilidades, volumen de ventas, complejidad del servicio, entre otras; además, se enfatiza en la forma en que el país utiliza el término "cicloturismo" y se plantean las elementos por considerar en la definición.
\end{abstract}

Palabras clave: Cicloturismo, Turismo en Bicicleta, Tour Operadores, Costa Rica.

Abstract

This research aims to describe the current situation of cycle tourism in Costa Rica. It is focused on the services that Costa Rican tour operators offer, taking into consideration significant characteristics such as strengths, weaknesses, sales, complexity of services, among others. Furthermore, the concept of "Cycle tourism" is pointed out to be present in the elements that might be valued in its conception.

Key Words: Bicycle tourism, Cycle tourism, Tour operators, Costa Rica.

\section{Introducción}

Al hablar de cicloturismo, las personas tienen la idea de realizar un recorrido enbicicleta por lugares de interés turístico, además de tener la sensación de bienestar físico y reducir la huella ecológica personal; características que son propias de la actividad son aprovechadas por los tour operadores para su respectivo mercadeo.

Al mismo tiempo, sí se toma en cuenta la gran cantidad de problemas ambientales, de salud y el hecho de que los turistas de hoy son más conscientes de sus accione, con niveles más altos de educación, es posible afirmar que el cicloturismo tiene una gran oportunidad de posicionarse dentro de las ofertas turísticas. Sin embargo, hablar de cicloturismo en Costa Rica es complicado, ya que actualmente no se manejan datos de esta actividad en el país; muchas personas no tienen conocimiento de la forma cómo desarrolla esta actividad en la región.

El Instituto Costarricense de Turismo (ICT), ente encargado de velar por la actividad turística
Al hablar de cicloturismo, las personas tienen la idea de realizar un recorrido enbicicleta por lugares de interés turístico, además de tener la sensación de bienestar físico y reducir la huella ecológica personal; características que son propias de la actividad son aprovechadas por los tour operadores para su respectivo mercadeo.

Al mismo tiempo, sí se toma en cuenta la gran cantidad de problemas ambientales, de salud y el hecho de que los turistas de hoy son más conscientes de sus accione, con niveles más altos de educación, es posible afirmar que el cicloturismo tiene una gran oportunidad de posicionarse dentro de las ofertas turísticas. Sin embargo, hablar de cicloturismo en Costa Rica es complicado, ya que actualmente no se manejan datos de esta actividad en el país; muchas personas no tienen conocimiento de la forma cómo desarrolla esta actividad en la región.

El Instituto Costarricense de Turismo (ICT), ente encargado de velar por la actividad turística

1 Bachiller en Turismo Ecológico y Licenciado en Gestión Ecoturística de la Universidad de Costa Rica. Gestor Ecoturístico Independiente. Correo electrónico: alucar888@gmail.com 
en general del país, divide estadísticamente las actividades turísticas en cuatro grandes grupos: ecoturismo, turismo de aventura, turismo náutico y turismo de bienestar, los cuales a su vez se subdividen en otras actividades, y en ninguna de las cuatro categoría se encuentra el cicloturismo, lo cual dificulta conseguir información de la actividad (ICT, 2014).

En cuanto a la formación de guías profesionales específicamente para esta actividad turística en el país, únicamente se ha encontrado una certificación otorgada por el Instituto Nacional de Aprendizaje (INA), instituto que para el año 2013 ofertó el servicio de capacitación a nivel técnico denominado: "Guía de turismo Aventura en Ciclismo de Montaña”, con una duración de 763 horas; no obstante, se debe aclarar que los aspirantes cursan un bloque común con todos los demás técnicos de aventura y solamente dos asignaturas los especializan en ciclismo de montaña, con una duración de 56 horas ,"Ajuste y Mantenimiento de Bicicletas y 50 horas Técnicas de Atención y Guiado en Travesías, Cabalgatas y Ciclismo de Montaña" (INA, 2013).

Asimismo, la licenciada Camacho, del Instituto Costarricense del Deporte y la Recreación (ICODER), menciona que a la fecha, la institución no tiene registrada ninguna asociación o federación relacionada con el cicloturismo o ciclismo recreativo. El accionar de la entidad está limitado a brindar asesoría en temas de logística de eventos deportivos y recreativos, si es solicitado por anticipado por comités, organizaciones y federaciones, por lo tanto, no cuentan con registros o información de la actividad ciclorturistica del país (R. Camacho, Comunicación Personal, 28 de septiembre del 2016). Esta circunstancias dejan gran cantidad de interrogantes sobre la situación real del cicloturismo costarricense, y la forma en que se está gestionando.

La falta de información cicloturística nacional justifica la presente investigación, con la cual se pretende recopilar información de los tour operadores encargados de ofertar y ejecutar la actividad en los diferentes sectores del país, y de esta forma brindar datos concisos de situación real de la actividad cicloturística en Costa Rica.

\section{Cicloturismo}

Actualmente, el cicloturismo es una actividad turística importante y practicada en una gran cantidad de países; sin embargo, cabe mencionar que es muy difícil establecer una definición, pues existen varias propuestas y varían según el autor, por esta razón, se considera necesario establecer un criterio acerca de los conceptos y enfoques desarrollados.

El diccionario en línea de la Real Academia Española (RAE) define el cicloturismo como el “Turismo que se practica en bicicleta., esta definición es muy general y no contempla una gran variedad de factores que se involucran en la actividad. Una definición más amplia la ofrecen Valencia y Ayón (2009), de la siguiente forma "El cicloturismo es una actividad de recreación en la cual, a través del ciclismo, se disfruta del esparcimiento y del conocimiento de un sitio nuevo. Este concepto ha sido definido por la tesista", mientras que Cox (2012), expone que el cicloturismo puede ser visto e interpretado desde diferentes perspectivas y puede presentar contrastes entre ocio y deporte, activo y pasivo, pero se debe mencionar que presenta un nicho de mercado emergente, ya que cambia algunas prácticas sociales, basado en los nuevos discursos de sostenibilidad y calidad de vida, además menciona que tiene una gran similitud con diferentes actividades (hiking, climbers y walkers) realizadas al aire libre y tienen en común el disfrute del paisaje y sus elementos.

Para Chang y Chang (2003), el cicloturismo es una actividad positiva que puede ser utilizada en dos sentidos: como medio de trasporte y como medio de recreación, además de que los cicloturistas son personas que utilizan las bicicletas para viajes de ocio y toman viajes relativamente cortos a baja velocidad. No obstante, Oliveira y Esperança (2011) presentan una definición más amigable con el medio, al considerar al cicloturismo parte del turismo sustentable, aseveran que ayuda a reducir la contaminación, el congestionamiento vial, así como beneficios en salud para los turistas que lo practican y en el ámbito local una activación de emprendimientos (negocios turísticos), protectores 
del ambiente y la cultura local. Se considera como un medio para redescubrir la cultura y naturaleza de los destinos por visitar, y el disfrute de experiencias orientadas al beneficio físico y psicológico de las personas.

Según Di Croce y Ferretty (2011), el cicloturismo se puede dividir en dos tipos diferentes: el primero llamado empresarial, que consiste en un paquete todo incluido ofertado por una empresa, en donde los participantes siguen un itinerario ya establecido y cuentan con guías capacitados para realizar los recorridos; mientras que el segundo corresponde al autogestionado, lo cual permite que los turistas decidan las actividades por realizar, para esto se refiere a "la estrategia de viaje", que permite a los turistas programar las decisiones de su viaje antes, durante y después de realizada las actividades. Por su parte, Lamont (2009) afirma que existen varios aspectos que deben ser incluidos en la definición de cicloturismo, entre los que sobresalen: el tiempo promedio de permanencia en el destino, la distancia óptima lejos del domicilio, observación de actividades o rutas ciclísticas, la utilización de la bicicleta como elemento fundamental del viaje, entre otros; las definiciones que consideren los aspectos mencionados serán definiciones más concretas y directas. Así es posible inferir que todas las definiciones expuestas anteriormente mencionan tres objetivos generales: el disfrute de la actividad, el desarrollo económico y ambiental de los destinos y por último el bienestar físico de los turistas.

Para el caso del territorio costarricense, es muy difícil conceptualizar la actividad cicloturistica, debido a la falta de información en el nivel general, sin embargo, se puede argumentar que el cicloturismo es percibido como una actividad más del turismo de aventura; un claro ejemplo de esta tendencia es reflejado por la información que brindan dos entidades estatales: el ICT (2014) y el INA (2013), ambas instituciones utilizan el término ciclismo de montaña como un sinónimo del cicloturismo, esta situación en particular limita el desarrollo de la actividad por las siguientes razones:
- al restarle importancia a la actividad no se tienen registros e información para desarrollar estudios de mercadeo, factibilidad de rutas, etc.,

- al limitar la actividad al ciclismo de montaña, no se consideran los tours realizados con baja intensidad y carentes de adrenalina, para descubrir atractivos naturales y culturas de las regiones visitadas, lo que se podría considerar en Costa Rica como nuevos segmentos de mercado; y

- se limita la implementación de una propuesta de formación en el nivel técnico de guías especializados en cicloturismo.

Las limitaciones mencionadas no deberían ser clasificadas como simples obstáculos, ya que son temas que se podrían traer a discusión académica y generar propuestas e información para el desarrollo del cicloturismo costarricense.

\section{Metodología}

Para la recolección de los datos se elaboró una lista de treinta y dos tour operadores que ofrecen la actividad cicloturística en el país. A cada tour operador se le envió una encuesta conformada por ocho preguntas vía correo electrónico. El objetivo de la encuesta es recolectar datos que reflejen la situación real de la actividad cicloturística del país, desde el punto de vista de los gestores; esta información es importante debido a que en Costa Rica no se han encontrado antecedentes académicos o datos generales sobre el tema.

Los resultados preliminares obtenidos son los siguientes:

- respondieron a la solicitud veintitrés tour operadores de treinta y dos; y

- nueve tour operadores son considerados como "No respondieron" a la solicitud, después de haber pedido su colaboración cuatro veces y no obtener respuesta.

De los veintitrés tour operadores que respondieron, se obtuvo información relevante para la investigación: 
- de los veintitrés tour operadores que respondieron a la solicitud, nueve respondieron el cuestionario enviado

- siete tour operadores mencionan que los tour de cicloturismo no los ejecuta el tour operador en sí, ya que son efectuados por otra empresa turística de las zonas del Arenal, Limón, Heredia y Guanacaste, la cual posee los recursos necesarios;

- $\quad$ seis tour operadores indican haber eliminado los tours de cicloturismo de la oferta turística, entre los motivos están la baja demanda, no era rentable para empresa y no se vendían; y

- un tour operador indica que no puede ayudar porque está manejando temas más importantes.

\section{Análisis de los resultados}

Dentro de las interrogantes se cuestionó cuál sería la principal razón para brindar la actividad cicloturística; los datos obtenidos muestran que de las empresas que actualmente están ofertando la actividad cicloturística lo hacen para aumentar o diversificar la oferta turística, además de utilizar a su favor las características de la zona en donde se comercializa el tour, al mismo tiempo se puede inferir que la experiencia en la gestión de la actividad no es importante para brindar el tour. La minoría de los tour operadores mencionan dedicarse a un segmento de mercado específico (Ver cuadro 1); sin embargo, para lograr una mejor interpretación de la información es necesario complementarla con un perfil del turista que adquiere los tour en bicicleta.

\section{Cuadro 1}

\section{Principal razón que motivó al tour operador a implementar el cicloturismo}

\begin{tabular}{lcc}
\hline Variables & Abssolutos & Relativos \% \\
\hline $\begin{array}{l}\text { Amplia experiencia de la } \\
\text { actividad }\end{array}$ & 3 & $33.33 \%$ \\
$\begin{array}{l}\text { Basado en el perfil turístico } \\
\text { que maneja la empresa }\end{array}$ & 2 & $22.23 \%$ \\
\hline Otra & 4 & $44.44 \%$ \\
\hline Total & 9 & $100 \%$ \\
\hline
\end{tabular}

Fuente: Elaboración propia, con datos de las encuestas aplicadas a tour operadores 2015.

Seguidamente, se consultó por los guías encargados de ejecutar los tour de cicloturismo, en espcífico si ellos cuentan con entrenamiento o capacitaciones idóneas para la actividad; un $67 \%$ de los tour operadores concuerdan en tener guías capacitados, sin embargo, es importante, mencionar que este dato puede variar debido a que en Costa Rica la legislación nacional exige a los guías una "Credencial de Guías" para ejercer y los faculta como personas capacitadas para el desarrollo de actividades turísticas, situación que puede ser utilizada por las empresas para generalizar la capacidad de los guías en diferentes actividades. Por el otro lado, un 33\% de las empresas reconoce la deficiencia en este aspecto y mencionan la realidad del país, por ejemplo: son proyectos en desarrollo por parte de la empresa y no existe una certificación para este tipo de turismo especializado en el país (Ver gráfico 1).

Después de conocer la situación de los guías, se consultó a las empresas si poseen un plan de emergencias o contingencias para ejecutar la 
actividad, esta información ayuda a entender mejor las capacitaciones de los guías y la complejidad del tour ofertado. Por las características propias de la actividad, un $78 \%$ de las empresas argumenta sí poseer una estrategia de emergencias: guías certificados en "Primeros auxilios y RCP", uso de botiquines portátiles, y los más complejos realizan un acompañamiento durante todo el recorrido con vehículo; mientras que un $28 \%$ no ha desarrollado un plan de contingencia y para las eventualidades se apoyan en póliza de viaje de los turistas (Ver gráfico 2).

\section{Gráfico 1}

\section{Capacitaciones de los guías para efectuar los tours}

¿Los guías cuentan con entrenamiento y/o capacitación para realizar tours de cicloturismo?

a sí no

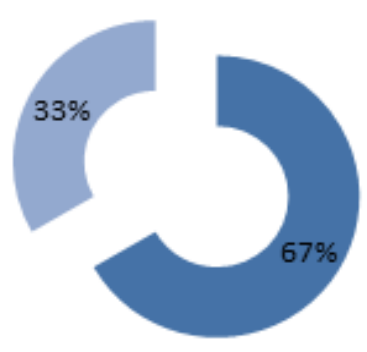

Fuente: Elaboración propia, con datos de las encuestas aplicadas a tour operadores 2015.

\section{Gráfico 2}

\section{Planes de emergencia o contingencia para la ejecución el tour}

¿El tour operador ha desarrollado un plan de emergencias o contingencias para la ejecución de la actividad?

घín sí no

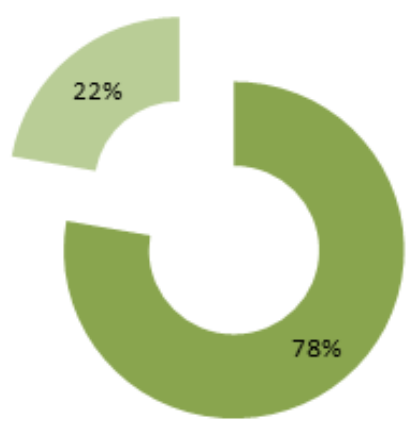


Una de las interrogantes del cicloturismo costarricense consiste en si es una actividad complementaria o una actividad más compleja. Con la finalidad de tener una noción más clara se les consultó a las empresas la duración, el 56\% realiza los tour en periodos de dos hasta seis horas en el mismo día, debido a la poca demanda y son considerados paseos recreativos de medio día, situación que refuerza la tendencia de considerar la actividad como una oferta complementaria a otras actividades turísticas en el destino, el restante $44 \%$ tienen capacidad de gestionar tours de varios días, pero deben ser solicitados con antelación por los turistas, debido a que no se ofertan por su poca demanda (Ver gráfico 3).

Para tener una percepción real de la demanda del cicloturismo en el territorio nacional, se les solicita a los tour operadores que identifiquen aproximadamente cuál sería el porcentaje de ventas de tour en bicicletas en comparación con el resto de servicios ofrecidos, las ventas son mínimas en la mayoría de las empresas, representadas con $77.77 \%$ de la muestra (Ver cuadro 2), la baja demanda de los tours podría considerarse una repercusión de la forma en que actualmente se gestiona la actividad, por ejemplo la publicidad, elemento del mercadeo empresarial que se podría ver restringido para el cicloturismo, específicamente al ser considerada una actividad de complemento en los destinos y ser simplemente una estrategia para diversificar la oferta turística.

\section{Gráfico 3}

\section{Duración los tours ofertados por los tour operadores}

\section{¿El tour operador gestiona tours de cicloturismo con una duración mayor de un día?}

sí $\square$ no

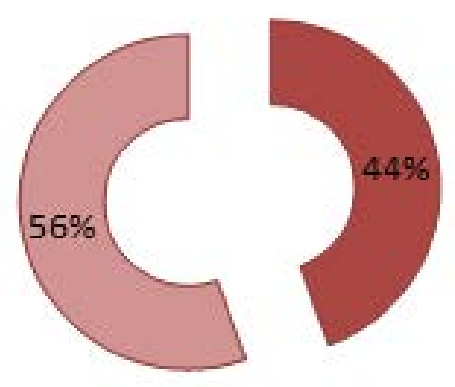




\section{Cuadro 2}

\section{Porcentaje aproximado de ventas de los tour de cicloturismo}

\begin{tabular}{lcc}
\hline Variables & Abssolutos & Relativos \% \\
\hline De 5\% a 20\% de las ventas & 7 & $77.77 \%$ \\
\hline De $81 \%$ a $100 \%$ de las ventas & 2 & $22.23 \%$ \\
\hline Total & 9 & $100 \%$ \\
\hline
\end{tabular}

Fuente: Elaboración propia, con datos de las encuestas aplicadas a tour operadores 2015.

Seguidamente, se le preguntó al tour operador cómo calificarían la actividad cicloturística del país con base en la experiencia, un $44.44 \%$ de la muestra la considera una actividad turística desarrollada, indicador que debe ser analizado en futuros trabajos académicos con mayor detalle, debido a que es contradictorio con la situación cicloturistica nacional (carencia de información en el nivel nacional, no se encuentran asociaciones inscritas en el ICODER, ausencia de guías especializados, entre otras). Podría pensarse que la respuesta es subjetiva por parte de los empresarios, basados en que se encuentran conformes con el margen de ventas de dicha actividad. Por otro lado, un $33.33 \%$ la considera apenas desarrollada y un $22.22 \%$ la piensa como actividad poco desarrollada (Ver cuadro 3), ambos porcentajes evidencian que algunas empresas perciben carencias en la gestión del cicloturismo nacional.

\section{Cuadro 3}

\section{Clasificación de la actividad cicloturística nacional, basada en la experiencia de los tour operadores}

\begin{tabular}{lcc}
\hline Variables & Abssolutos & Relativos \% \\
\hline Poco desarrollada & 2 & $22.23 \%$ \\
\hline Apenas desarrollada & 3 & $33.33 \%$ \\
Desarrollada & 4 & $44.44 \%$ \\
\hline Total & 9 & $100 \%$ \\
\hline
\end{tabular}


En el nivel de empresa, se les consultó sobre una fortaleza percibida al brindar tours de cicloturismo, seguidamente se transcriben las repuestas a la interrogante sin ninguna alteración, con respeto de la expresión de los empresarios:

- conocimiento de la zona;

- un mecanismo para captar clientes interesados en este tipo de actividad y así aumentar ganancias;

- conocimiento de la disciplina;

- el ciclismo es un área de mucho auge por lo que hay mucha demanda de este tipo de servicio;

- atraer otro tipo de turismo, bajo costo operacional y avistamiento de áreas circunvecinas a través de una actividad adicional a los tours ya existentes;

- calidad del servicio.

- amplia experiencia en el ciclismo como deporte y recreación, nos hacen diseñar un producto adecuado para el perfil del turista que busca este tipo de experiencia de aventura con el factor físico de desempeño implícito; y

- ofrece al turista una opción amigable con el medio ambiente, justamente hacia esa dirección se orientan los requerimientos actuales de los consumidores.

La percepción tenida por los empresarios turísticos sobre las fortalezas de ofrecer tours de cicloturismo es muy lineal o superficial, orientada a mayores beneficios económicos, variedad de servicios ofertados y la atracción de segmentos especializados de turistas (actividades amigables con el ambiente). Por otro lado, las debilidades percibidas por la empresa al brindar dicho servicio turístico se transcriben a continuación:

- renovación del equipo que se usa;

- en nuestro caso ninguno, si el cliente lo quiere se le ofrece, pero no se registra ningún tipo de problema o debilidad;

- pocas alternativas profesionales para la práctica;
- el desconocimiento de las empresas pequeñas, a la gente no le genera mucha confianza el no contar con una larga trayectoria;

- el riesgo de accidentes o daño del equipo o bicicletas, cascos, etc.;

- precios de los productos un poco más altos, comparados con otros países de la región, en cuanto a turismo;

- en este momento la única debilidad es la limitante en capacidad de atención de volumen;

- El turista nacional y extranjero piensan que la actividad es muy fuerte para el clima del área; y

- No identifico un problema, considero con respecto a la experiencia personal que es un mercado en crecimiento.

Las debilidades planteadas son problemas en el nivel administrativo, de logística o publicidad, que se esperarían que sean identificadas por lo empresarios; no obstante, la debilidad más recurrente es el gasto económico realizado por la compra y mantenimiento del equipo, y en algunos casos no se identificó ninguna debilidad.

\section{Conclusiones}

Durante el proceso de recopilación de datos, no se encontró ningún estudio de cicloturismo para Costa Rica, por lo cual, con el presente estudio se logró obtener una gran cantidad de información que permite visualizar más claramente el estado real de la actividad cicloturística en el país.

El cicloturismo en Costa Rica es una actividad turística poco desarrollada y analizada, esta situación se debe a que son muy pocos los tour operadores que ofrecen la actividad a los turistas nacionales y extranjeros. Al mismo tiempo, la actividad cicloturística nacional carece de un seguimiento por parte de las entidades estatales (ICT, INA, ICODER), instituciones que podrían ayudar al desarrollo, posicionamiento y recopilación de datos en el nivel nacional e internacional. 
Es importante aclarar una situación en particular: la mayoría de los tour operadores que ofertan este tipo de tour en bicicleta, no lo realizan ellos mismos, la ejecución de dichos tours recae en unos pocos tour operadores que cuentan con los recursos para desarrollar la actividad.

Con base en la información recopilada por medio de la encuesta a los tour operadores, se han identificado tres zonas importantes para el desarrollo de la actividad y al mismo tiempo son los lugares donde se concentran los tour operadores con los recursos para realizar los tour de cicloturismo, donde el más importante es la zona que rodea al Volcán Arenal, seguido de Guanacaste y por último la zona atlántica. Asimismo, al considerar la experiencia de los tour operadores, la actividad cicloturística en algunos casos se ha pensado en la oferta turística de las empresas con la finalidad de ofrecer más servicios o variedad a los turistas, también se mencionó casos en los cuales se eliminó la actividad cicloturística por no tener demanda de los turistas o no ser rentable para la empresa.

Sin embargo, los tour operadores utilizan las características principales de la actividad cicloturística como soncuidar el medio y mejorar la salud de las personas participantes, para mercadear los servicios ofrecidos y de esta forma atraer un nicho de mercado específico. De la misma manera, algunos tour operadores no manejan el concepto "cicloturismo", los tours en bicicleta vendidos a los turistas están clasificados en actividades de un día, esta situación puede afectar negativamente al desarrollo de la actividad en el país, al no proporcionarle el adecuado seguimiento y, en consecuencia, la información de la actividad será muy difícil de conseguir. De acuerdo con la información de los tour operadores y la escasa información de las entidades nacionales (ICT, INA), en Costa Rica el término "cicloturismo" es considerado un sinónimo de ciclismo de montaña, actividad que pertenece al turismo de aventura, como lo serían el rafting y tirolesa.

Todavía falta mucha información de la actividad cicloturística costarricense, es un tema poco explorado académicamente y debe abordarse por diferentes perspectivas, de esta forma se pueden generar datos y discusiones académicas valiosas con el fin de mejorar el desarrollo de esta actividad en el país.

\section{Reflexiones finales}

La debilidad más recurrente identificada por las empresas es la compra y mantenimiento de los equipos necesarios para la actividad, una estrategia para mitigar este problema sería la realización de un estudio económico que demuestre la viabilidad de ejecutar un plan de compras y mantenimiento de equipos, con la designación de un presupuesto, acciones por desarrollar y encargados de su ejecución.

La creación de cluster o encadenamiento turístico con negocios ubicados en las rutas seleccionadas (generación de empleos indirectos, publicidad) y la responsabilidad social y ambiental de la empresa (certificaciones, CST) podrían ser importantes fortalezas desconocidas actualmente por los tour operadores, ambas propuestas ayudarían a mejorar la gestión actual de la actividad.

Una propuesta para dar a conocer el cicloturismo en Costa Rica, sin la dependencia de los tour operadores, es la creación de asociaciones de cicloturismo formalmente inscritas ante el Instituto Costarricense del Deporte y la Recreación (ICODER), además, la entidad regida por la ley 7800 , en su capítulo II, artículo 20, promueve programas de recreación y salud, condiciones implícitas en el cicloturismo. Asimismo, es importante mencionar que las asociaciones por formar serian no lucrativas, por consiguiente, el ingreso indirecto que podrían percibir las comunidades, cuando la actividad es organizada por empresas turísticas, pasaría a hacer directo, lo cual sería un incentivo para la creación de las asociaciones o algún tipo de emprendedurismo comunal.

La presente investigación demostró que la actividad cicloturistica nacional recae sobre unos pocos tour operadores; sin embargo, la creación de una alianza empresarial podría beneficiar la actividad; un grupo organizado con los mismos 
intereses podrían solicitar el apoyo del Instituto Costarricense de Turismo (ICT), en mercadeo turístico (espacios en ferias turísticas), al mismo tiempo, realizar una solicitud formal al Instituto Nacional de Aprendizaje (INA) para la incorporación de un plan de estudios especializado en cicloturismo y prometer las instalaciones de las empresas para realización de prácticas supervisadas. 


\section{Bibliografía}

Asamblea Legislativa. (1998). Ley Creación el Instituto del Deporte y Recreación $N^{\circ} 7800$. San José, Costa Rica. Recuperado de: http:// www.pgrweb.go.cr/scij/Busqueda/Normativa/ Normas/nrm_texto_completo.aspx?param $1=$ NRTC\&nValor $1=1 \&$ nValor2 $=26290 \&$ nValor 3=78197\&strTipM=TC

Chang y Chang. (2003). "A strategic study of bicycle tourism in Taiwan". Journal of the Eastern Asia Society for Transportation Studies, 5, 1675-1685. Recuperado de http://www.easts. info/2003journal/papers/1675.pdf

Di Croce y Ferretty. (2011). Cicloturismo: un abordaje desde el concepto estrategia de viaje. IX Congreso Argentino y IV Latinoamericano de Educación Física y Ciencias. Facultad de Humanidades y Ciencias de la Educación, Universidad de la Plata, Argentina. Recuperado de http://hdl.handle.net/10915/35352

Instituto Costarricense de Turismo. (2014). Estimación del porcentaje de turistas que realizaron las siguientes actividades Vía Aérea 2009-2013. Recuperado de http://www. visitecostarica.com

Instituto Nacional de Aprendizaje. (2013). Oferta curricular subsector servicios turísticos 2013. Recuperado de http://www.ina.ac.cr

Lamont, MJ. (2009). Independent bicycle tourism in Australia: a whole tourism systems analysis. PhD thesis. Southern Cross University, Lismore, NSW. Recuperado de http://epubs. scu.edu.au/cgi/viewcontent.cgi? article $=1097$ \&context=theses

Oliveira y Esperança. (2011). "Bike usage and cycle tourism: the pattern of portuguese associated bike riders". Encontros Científicos - Tourism y Management Studies, 191-200. Recuperado de http://dialnet.unirioja.es/descarga/ articulo/5018484.pdf
Peter Cox (2012). "Strategies Promoting Cycle Tourism in Belgium: Practices and Implications, Tourism Planning and Development". 9:1, 2539, DOI: 10.1080/21568316.2012.658167

Real Academia Española. (2015). Cicloturismo. Diccionario de la Lengua Española [versión electrónica]. España: Real Academia Española., http://www.rae.es

Valencia \& Ayón (2009). El Cicloturismo y su aporte a la oferta turística de la ruta: Valdivia - Sinchal - Barcelona - Loma Alta - Bambil Collao Colonche - Barbascal - Palmar. FIMCBOR. Recuperado de http://www.dspace.espol.edu. ec/xmlui/handle/123456789/1715 